\title{
Arf6-GEF BRAG1 Regulates JNK-Mediated Synaptic Removal of GluA1-Containing AMPA Receptors: A New Mechanism for Nonsyndromic X-Linked Mental Disorder
}

\author{
Kenneth R. Myers, ${ }^{1 \star}$ Guangfu Wang, ${ }^{2 \star}$ Yanghui Sheng, ${ }^{2,4}$ Kathryn K. Conger, ${ }^{1}$ James E. Casanova, ${ }^{1,2}$ and J. Julius Zhu ${ }^{2,3}$ \\ Departments of ${ }^{1}$ Cell Biology, ${ }^{2}$ Pharmacology, and ${ }^{3}$ Neuroscience, University of Virginia Health System, Charlottesville, Virginia $22908-0732$ and ${ }^{4}$ Yuanpei \\ College, Peking University, Beijing, 100871, China
}

\begin{abstract}
Activity-dependent modifications of excitatory synapses contribute to synaptic maturation and plasticity, and are critical for learning and memory. Consequently, impairments in synapse formation or synaptic transmission are thought to be responsible for several types of mental disabilities. BRAG1 is a guanine nucleotide exchange factor for the small GTP-binding protein Arf6 that localizes to the postsynaptic density of excitatory synapses. Mutations in BRAG1 have been identified in families with X-linked intellectual disability (XLID). These mutations mapped to either the catalytic domain or an IQ-like motif; however, the pathophysiological basis of these mutations remains unknown. Here, we show that the BRAG1 IQ motif binds apo-calmodulin (CaM), and that calcium-induced CaM release triggers a reversible conformational change in human BRAG1. We demonstrate that BRAG1 activity, stimulated by activation of NMDA-sensitive glutamate receptors, depresses AMPA receptor (AMPA-R)-mediated transmission via JNK-mediated synaptic removal of GluA1-containing AMPA-Rs in rat hippocampal neurons. Importantly, a BRAG1 mutant that fails to activate Arf6 also fails to depress AMPA-R signaling, indicating that Arf6 activity is necessary for this process. Conversely, a mutation in the BRAG1 IQ-like motif that impairs CaM binding results in hyperactivation of Arf6 signaling and constitutive depression of AMPA transmission. Our findings reveal a role for BRAG1 in response to neuronal activity with possible clinical relevance to nonsyndromic XLID.
\end{abstract}

\section{Introduction}

The majority of fast excitatory synaptic transmission in the CNS is mediated by AMPA- and NMDA-type ionotropic glutamate receptors (Traynelis et al., 2010). A key factor underlying the strength of individual excitatory synapses is the number of AMPA receptors (AMPA-Rs) at synapses, which is tightly regulated by AMPA-R trafficking. This regulated trafficking, largely mediated by NMDA-R signaling, plays a key role in both synaptic transmission and plasticity (Kerchner and Nicoll, 2008; Kessels and Malinow, 2009; Anggono and Huganir, 2012). Both hyporegulation and hyperregulation of synaptic AMPA-R trafficking reduce the capacity of synaptic plasticity (McCormack et al., 2006), and are thought to underlie numerous cognitive disorders, including mental retardation (Costa and Silva, 2003; Thomas and Huganir, 2004; Stornetta and Zhu, 2011).

The ADP-ribosylation factor (Arf) proteins are a family of six small, ubiquitously expressed GTP-binding proteins (Donaldson

\footnotetext{
Received April 20, 2012; revised June 21, 2012; accepted July 2, 2012.

Author contributions: K.R.M., G.W., Y.S., J.E.C., and J.J.Z. designed research; K.R.M., G.W., Y.S., and K.K.C. performed research; K.R.M., G.W., Y.S., K.K.C., J.E.C., and J.J.Z. analyzed data; K.R.M., G.W., Y.S., J.E.C., and J.J.Z. wrote the paper.

This work was supported by National Institutes of Health Grant GM078585 (J.E.C.). We thank Bettina Winckler and David Castle for careful reading of the manuscript.

*K.R.M. and G.W. contributed equally to this work.

The authors declare no competing financial interests.

Correspondence should be addressed to Dr. James E. Casanova, University of Virginia, Department of Cell Biology, P0 Box 800732, Jordan Hall 3-59, Charlottesville, VA 22908-0732. E-mail: jec9e@virginia.edu.

DOI:10.1523/JNEUROSCI.1942-12.2012

Copyright $\odot 2012$ the authors $\quad 0270-6474 / 12 / 3211716-11 \$ 15.00 / 0$
}

and Jackson, 2011). Of these, Arf6 localizes primarily to the plasma membrane/endosomal system, and is best known as a regulator of endocytic trafficking and actin cytoskeleton dynamics (D'Souza-Schorey and Chavrier, 2006; Myers and Casanova, 2008). In hippocampal neurons, Arf6 has been shown to regulate dendritic arborization (Hernández-Deviez et al., 2002), axonal outgrowth (Hernández-Deviez et al., 2004), dendritic spine formation (Miyazaki et al., 2005; Choi et al., 2006), and the assembly of clathrin/AP2 complexes at synaptic membranes (Krauss et al., 2003). The human genome contains 15 Arf-guanine nucleotide exchange factors (GEFs), which catalyze the exchange of GDP for GTP via the evolutionarily conserved catalytic Sec7 domain (Casanova, 2007). The brefeldin-resistant Arf-GEFs (BRAGs) comprise a subfamily of three proteins that are abundantly expressed within the postsynaptic density (PSD; Jordan et al., 2004; Peng et al., 2004; Dosemeci et al., 2007).

BRAG2/IQSec1 has recently been shown to interact directly with the cytoplasmic domain of the AMPA-R subunit GluA2 and to regulate its synaptic activity-dependent endocytosis (Scholz et al., 2010). In contrast, BRAG1/IQSec2 is reported to interact with NMDA-Rs, but not AMPA-Rs, through an indirect mechanism involving the synaptic scaffolding protein PSD-95 (Sakagami et al., 2008). Recently, Shoubridge et al. (2010) identified four nonsynonymous single nucleotide polymorphisms (SNPs) in BRAG1 from families with nonsyndromic X-linked intellectual disability (XLID). Three of these SNPs led to nonconserved amino acid substitutions within the catalytic Sec7 domain, while the fourth was a 
A

\begin{tabular}{|c|c|c|c|c|c|}
\hline BRAG2 & IQ & Sec7 & $\mathrm{PH}$ & CC & PDZ \\
\hline BRAG3 & $\mathrm{Cc}$ & Sec7 & $\mathrm{PH}$ & CC & DDZ \\
\hline
\end{tabular}

B

$\begin{array}{ll}\text { IQ-like motif } & \text { [FILV] } Q \times x x[R K] \times x x x \\ \text { BRAGI-WT } & \text { IQTAFRQYRM } \\ \text { BRAGI-IQ } & \text { AATAFAQYRM }\end{array}$

C

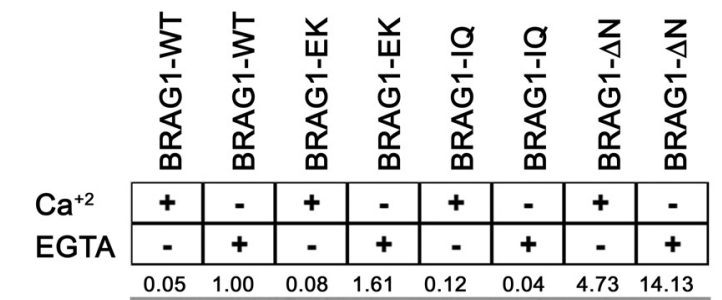

CaM-Seph IP WB: Myc

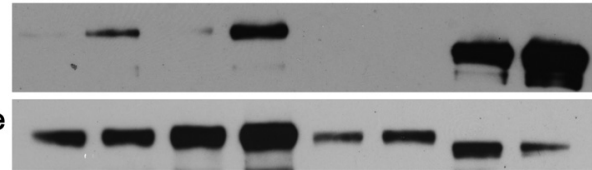

Figure 1. BRAG1 binds $\mathrm{Ca}^{2+}$-free calmodulin. $\boldsymbol{A}$, Domain organization of the three BRAG family members. $\boldsymbol{B}$, Consensus sequence for IQ motifs, compared with the IQ-like motif in wild-type BRAG1. The bottom sequence shows mutations introduced to generate BRAG1-IQ. C, CaM binding to wild-type and mutant BRAG1. HeLa cells were transiently transfected with myc-tagged BRAG1-WT, BRAG1-EK, BRAG1-IQ, or BRAG1- $\triangle \mathrm{N}$ and lysed in the presence of either $2 \mathrm{~mm} \mathrm{EGTA}$ or $2 \mathrm{~mm} \mathrm{CaCl}$. Cell lysates were then incubated with CaM-Sepharose, and BRAG1 binding was analyzed by anti-myc immunoblotting. Binding was quantified by densitometry. The signal for BRAG1-WT was normalized to a value of 1 and relative binding values are shown. The blot shown is representative of three independent experiments.

nonconserved substitution within an IQ motif (Shoubridge et al., 2010).

Here we report that BRAG1 has an integral role in synaptic transmission. We show that expression of exogenous BRAG1 in CA1 hippocampal neurons results in depression of AMPA-R-mediated synaptic transmission, in a manner dependent upon upstream NMDA-R activation. This depression is also dependent upon BRAG1 catalytic activity, indicating that it requires Arf6 activation. We show that BRAG1 binds calmodulin (CaM), and that a mutation in the IQ motif that prevents CaM binding results in constitutive (NMDA-R-independent) depression of AMPA-Rmediated transmission. Furthermore, BRAG1 appears to selectively control the trafficking of GluA1-containing AMPA-Rs by stimulating JNK signaling. Together, these results indicate that BRAG1 acts as a CaM-responsive switch to control AMPA-R signaling downstream of NMDA-R activation.

\section{Materials and Methods}

Reagents and antibodies. The reagents used in this study include ionomycin (Invitrogen I-24222), NMDA (Sigma M3262), APV (Sigma A5282), BAPTA-AM (Invitrogen B-1205), and CaM-Sepharose 4B (GE Life Sciences). Primary antibodies used were 9E10 $\alpha$-Myc, $16 \mathrm{~B} 12 \alpha$-HA (Covance), $\alpha$-GFP (Invitrogen), and $\alpha$-PSD-95 (ThermoFisher Scientific). BRAG1 rabbit antiserum was raised against a peptide, corresponding to amino acids 258-275 (CAVDSPGSQPPYRLSQLP), coupled to keyhole limpet hemocyanin as antigen.

DNA constructs. Human BRAG1 (KIAA0522) cDNA was obtained from the Kasuza DNA Research Institute. The coding sequence of BRAG1 was subcloned into pCMV3A-Myc using HindIII/XhoI. The BRAG1-E849K and BRAG1-IQ mutants were made by site-directed mutagenesis. The BRAG1- $\Delta \mathrm{N}$ mutant was made by digesting BRAG1-WT with EcoRV/NruI, which creates an in-frame deletion of the $\mathrm{N}$-terminal
213 aa. To create Cherry-tagged versions, BRAG1 was digested out of pCMV3A-Myc using HindIII/XhoI and ligated into mCherry-C2 (Clontech) using HindIII/SalI. The BRAG1mCherry fusions were digested out of the mCherry-C2 plasmid using NheI/XbaI and ligated into pSinRep5 using XbaI to make Sindbis virus constructs.

Cell culture. HeLa cells were cultured and transfected as described previously (Santy and Casanova, 2001). Dissociated hippocampal neuron cultures were prepared and transfected as described by (Zhang et al., 2003). For ionomycin stimulation, HeLa cells were switched 4-6 h post-transfection into serum-free DMEM for 16-20 additional hours. Cells were then moved into fresh phenol red-free, serumfree DMEM (plus 25 mM HEPES for time-lapse imaging) containing either dimethylsulfoxide (DMSO) vehicle or $5 \mu \mathrm{M}$ ionomycin for $3 \mathrm{~min}$.

Pulldown assays. Arf6-GTP levels were measured using a GST-GGA3 pulldown assay as described previously (Santy and Casanova, 2001). Results are reported as mean \pm SEM and statistical differences were determined using the Wilcoxon matched pairs test. For CaM binding, HeLa cells transiently expressing myctagged BRAG1 constructs were lysed on ice in buffer A (150 mm NaCl, 1\% Triton X-100, 50 mM Tris, protease inhibitors; $\mathrm{pH}$ 7.4) containing either $1 \mathrm{~mm} \mathrm{CaCl}$ or $1 \mathrm{~mm}$ EGTA, and incubated with CaM-Sepharose for $2 \mathrm{~h}$. Densitometry was performed using ImageJ Software (National Institutes of Health) to quantify protein expression levels.

Immunofluorescence microscopy. HeLa cells were grown on glass coverslips, fixed, and processed for microscopy as described in (Santy and Casanova, 2001). Fixed images were acquired using a $60 \times$ objective on a Nikon Eclipse E800 microscope and a Q-Imaging Retiga CCD camera. Live cells were imaged in extracellular solution ( $150 \mathrm{~mm} \mathrm{NaCl}, 10 \mathrm{~mm}$ HEPES, $5 \mathrm{~mm} \mathrm{KCl}$, and $30 \mathrm{~mm}$ glucose; $\mathrm{pH}$ 7.4) with or without $2 \mathrm{~mm} \mathrm{CaCl}_{2}$ using a $60 \times$ objective on a DeltaVision deconvolution microscope (Applied Precision). For quantitation of BRAG1 condensation, NIS-Elements software (Nikon) was used to set a defined background threshold and automatically count puncta up to $2 \mu \mathrm{m}^{2}$.

Hippocampal slice cultures. Cultured rat hippocampal slices were prepared from postnatal 6- to 7-d-old rats of either sex, infected with Sindbis virus after 7-14 $\mathrm{d}$ in vitro (DIV) to deliver recombinant proteins into CA1 pyramidal neurons as described previously (Qin et al., 2005; McCormack et al., 2006). Hippocampal extracts were prepared by homogenizing hippocampal CA1 regions isolated from cultured slices (Zhu et al., 2000, 2002), Viral expression efficacy of recombinant proteins in these experiments was high ( $>95 \%$ of CA1 neurons). Homogenizing solution contained the following (in mM or percentage): 10 HEPES, 150 $\mathrm{NaCl}, 10$ EDTA, 4 EGTA, 0.2 PMSF, 0.1 NaPPi, 0.5 NaF, $1 \mathrm{Na}_{3} \mathrm{VO}_{4}$, and Triton 1\%. Membranes were blotted with anti-phospho-JNK (1:2000; Cell Signaling Technology) and anti-phospho-p38 MAPK antibody (1: 2000;Cell Signaling Technology), stripped and reblotted with anti-JNK (1:2000;Cell Signaling Technology) and anti-p38 MAPK antibody (1: 1000;Cell Signaling Technology). Western blots were quantified by chemiluminescence and densitometric scanning of the films under linear exposure conditions. The dendritic and spine expression of mCherryBRAG1 was imaged with a custom-made two-photon laser scanning microscope (Zhu et al., 2000; Kolleker et al., 2003).

Electrophysiology. Simultaneous whole-cell recordings were obtained from nearby infected and noninfected neuron pairs, under visual guidance using fluorescence and transmitted light illumination with two Axopatch-200B amplifiers (Molecular Devices) as previously described 

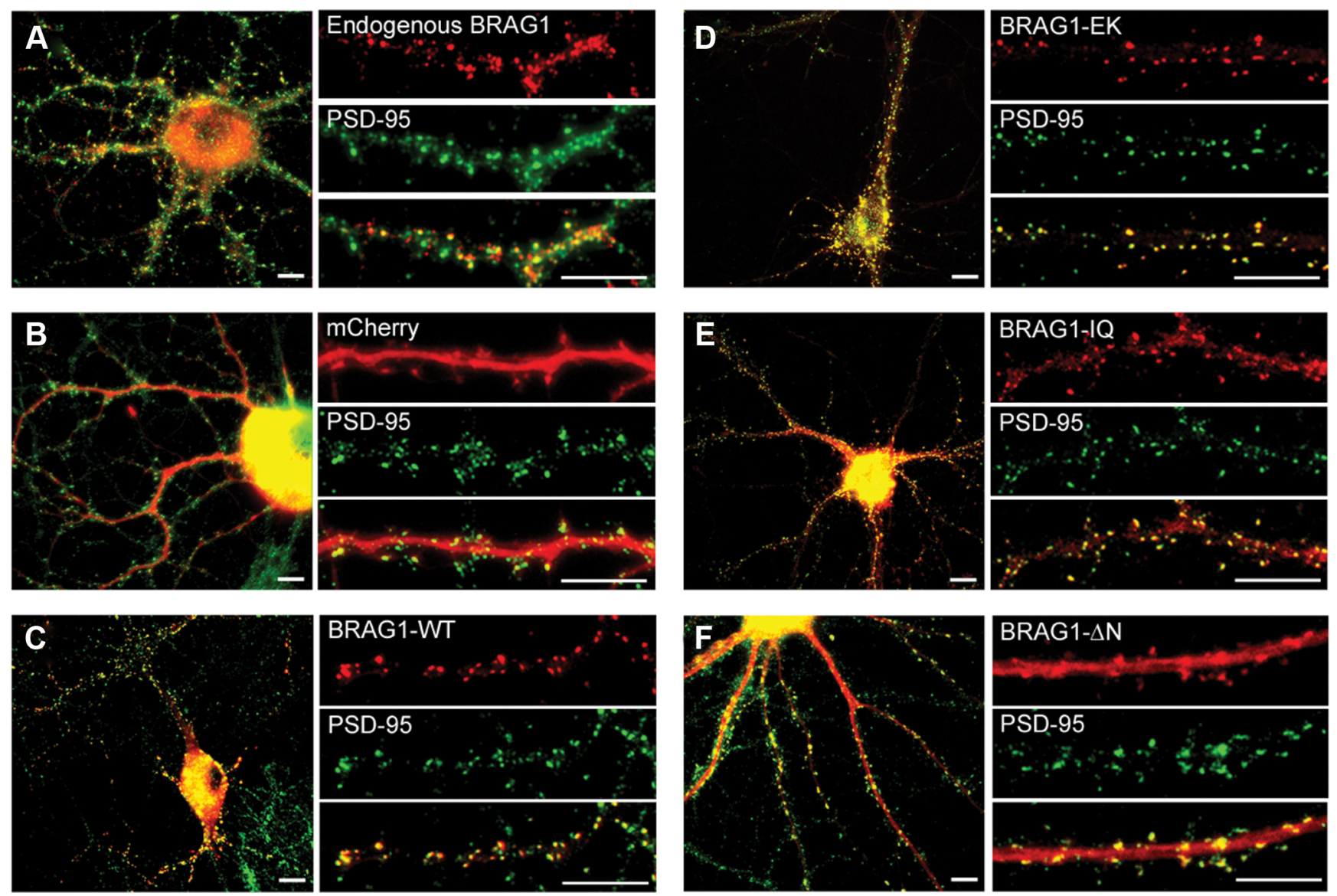

Figure 2. BRAG1 mutants localize to excitatory synapses in hippocampal neurons. $A$, Dissociated rat hippocampal neurons were fixed at DIV 21, and coimmunostained with rabbit polyclonal anti-BRAG1 and mouse anti-PSD-95 antibodies. $\boldsymbol{B}-\boldsymbol{F}$, Hippocampal neurons were transfected (DIV 6) with the indicated mCherry-tagged constructs and fixed (DIV 21) for epifluorescence imaging. Cells expressing mCherry $(\boldsymbol{B})$, mCherry-BRAG1-WT $(\boldsymbol{C})$, mCherry-BRAG1-EK $(\boldsymbol{D})$, mCherry-BRAG1-IQ $(\boldsymbol{E})$, and mCherry-BRAG1- $\Delta \mathrm{N}(\boldsymbol{F})$ were costained with PSD-95 antibody to detect excitatory synapses. Scale bars: $10 \mu \mathrm{m}$.

(Qin et al., 2005; McCormack et al., 2006; Zhu, 2009). Cultured rat organotypic slices exhibit relatively high spontaneous activity comparable to intact brains. Thus, high calcium and magnesium bath solution $\left(29 \pm 1.5^{\circ} \mathrm{C}\right)$, containing the following (in $\mathrm{mm}$ ): $119 \mathrm{NaCl}, 2.5 \mathrm{KCl}, 4$ $\mathrm{CaCl}_{2}, 4 \mathrm{MgCl}_{2}, 26 \mathrm{NaHCO}_{3}, 1 \mathrm{NaH}_{2} \mathrm{PO}_{4}, 11$ glucose, 0.1 picrotoxin, and 0.002 2-chloroadenosine, $\mathrm{pH}$ 7.4, was used to prevent epileptic activity after blocking inhibition. The bath solutions were gassed with $5 \% \mathrm{CO}_{2} /$ $95 \% \mathrm{O}_{2}$. Patch recording pipettes $(3-6 \mathrm{M} \Omega$ ) contained the following (in $\mathrm{mM}$ ): 115 cesium methanesulfonate, $20 \mathrm{CsCl}, 10$ HEPES, $2.5 \mathrm{MgCl}_{2}, 4$ $\mathrm{Na}_{2} \mathrm{ATP}, 0.4 \mathrm{Na}_{3} \mathrm{GTP}, 10$ sodium phosphocreatine, 0.6 EGTA, and 0.1 spermine, at $\mathrm{pH}$ 7.25. Synaptic responses were evoked by bipolar electrodes with single voltage pulses $(200 \mu$ s, up to $20 \mathrm{~V})$ placed in hippocampal stratum radiatum $\sim 300 \mu \mathrm{m}$ away from the recorded hippocampal CA1 pyramidal neurons. To minimize the effect from AMPA responses, the peak NMDA responses at $+40 \mathrm{mV}$ were measured after digital subtraction of estimated AMPA responses at $+40 \mathrm{mV}$. Results are reported as mean \pm SEM and statistical differences were determined using Wilcoxon test.

\section{Results}

\section{BRAG1 interaction with CaM}

IQ motifs are best known as binding domains for CaM. Although BRAG1, BRAG2, and BRAG3 each contain an IQ-like motif $\mathrm{N}$ terminal to the catalytic domain (Fig. 1A), it has not yet been demonstrated that any of the BRAGs do indeed bind CaM. Inspection of this motif (residues 354-363) indicated that it fits the consensus sequence for calcium-independent CaM binding (Fig. 1B) (Rhoads and Friedberg, 1997). To determine whether this is the case, lysates of HeLa cells expressing Myc-tagged BRAG1 were incubated with CaM-Sepharose in either the presence or absence of $\mathrm{Ca}^{2+}$ ( $2 \mathrm{mM} \mathrm{CaCl}_{2}$ or $2 \mathrm{~mm}$ EGTA, respectively). As shown in Figure $1 C$, BRAG1 was robustly precipitated by CaM-Sepharose, but not Sepharose alone. Moreover, this interaction was strengthened in the presence of EGTA, indicating that BRAG1 preferentially binds to $\mathrm{Ca}^{2+}$-free CaM. Substitution of three conserved residues within the consensus IQ motif ( $\mathrm{I}^{354} \mathrm{QTAFR}$ to AATAFA, hereafter referred to as BRAG1-IQ) completely abrogated CaM binding (Fig. 1C). However, mutation of a conserved glutamate residue within the Sec7 domain essential for catalytic activity (E849K, hereafter referred to as BRAG1-EK) (Shoubridge et al., 2010), had no effect on the ability of BRAG1 to bind CaM, indicating that catalytic activity does not impact CaM binding (Fig. 1C). Deletion of an N-terminal coiled-coil domain (BRAG1- $\Delta N$ ) does appear to result in more efficient CaM binding than BRAG1-WT. This may be a result of the enhanced solubility of BRAG1- $\Delta \mathrm{N}$ (see below), or it could suggest that the coiled-coil motif regulates accessibility of the IQ motif to CaM.

\section{BRAG1 localization to the PSD}

Previous studies have revealed the localization of BRAG1 specifically at the postsynaptic membrane of excitatory synapses using both immunofluorescence and electron microscopy (Murphy et al., 2006; Sakagami et al., 2008). To confirm this localization, we stained dissociated rat hippocampal neurons at 21 DIV with rab- 
A

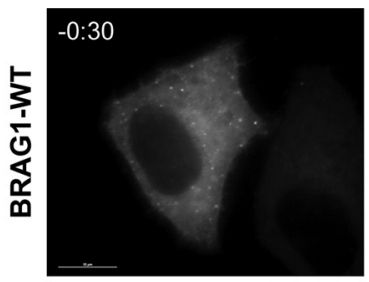

B

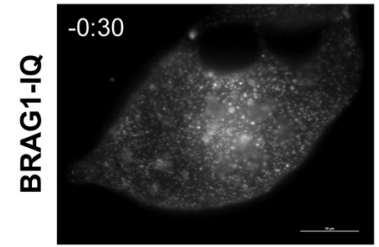

C
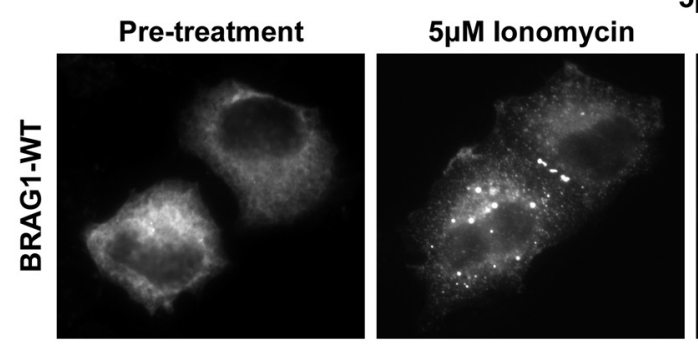

$5 \mu \mathrm{M}$ lonomycin + washed $+5 \mathrm{mM}$ BAPTA-AM

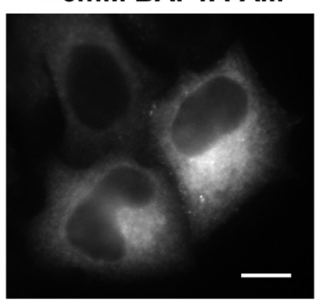

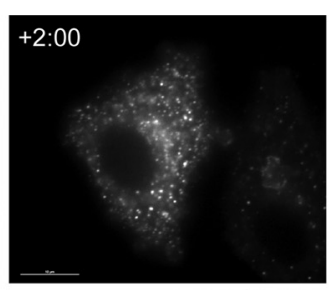

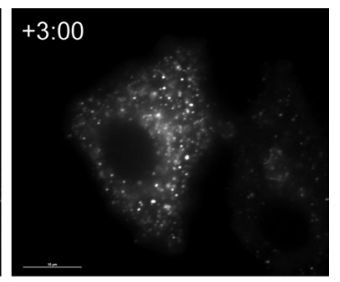

D
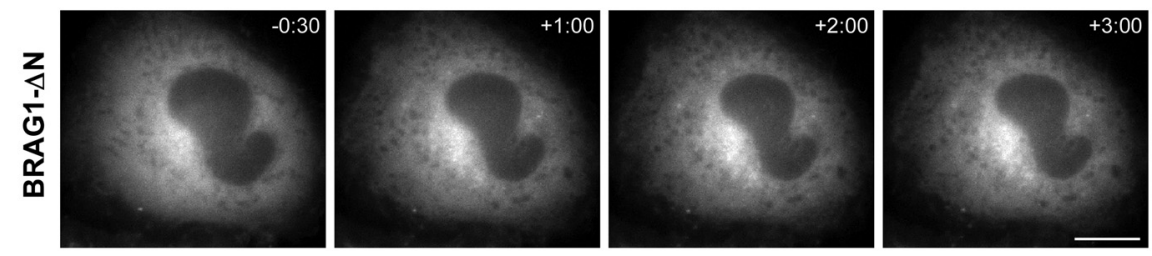

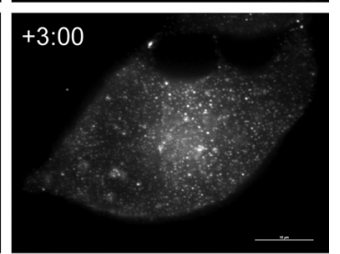

$\mathbf{E}$

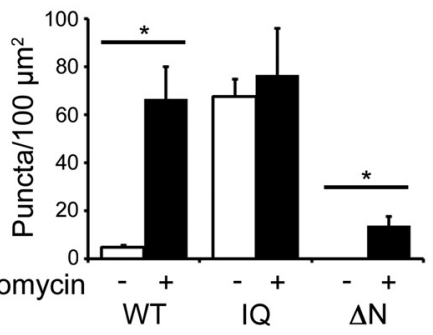

$\mathbf{F}$

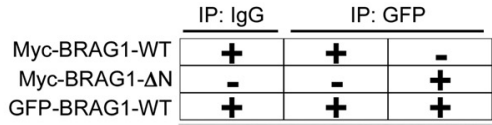

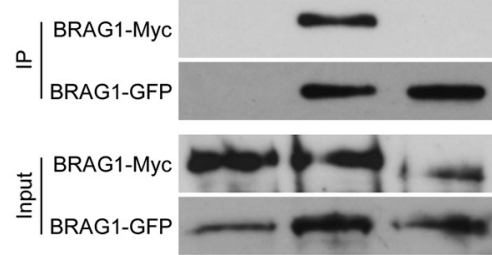

Figure 3. $\mathrm{Ca}^{2+}$ influx triggers a reversible conformational change in BRAG1. $A, B$, Formation of cytoplasmic puncta in response to calcium influx. HeLa cells expressing mCherry-BRAG1-WT ( $\boldsymbol{A}$ ) or mCherry-BRAG1-IQ $(\boldsymbol{B})$ were stimulated with $5 \mu \mathrm{m}$ ionomycin for $3 \mathrm{~min}$. Single frames from time-lapse movies beginning at $30 \mathrm{~s}$ before ionomycin treatment $(-30)$ are shown. Note that BRAG1-IQ is punctate in the absence of ionomycin and does not change its distribution after ionomycin addition. C, Calcium-induced condensation of BRAG1 is reversible. HeLa cells were either left untreated (left), were treated with $5 \mu \mathrm{m}$ ionomycin for $3 \mathrm{~min}$ (center), or were treated with $5 \mu \mathrm{m}$ ionomycin for $3 \mathrm{~min}$ followed by incubation with $5 \mathrm{~mm}$ BAPTA-AM for $10 \mathrm{~min}$ (right). D, BRAG1- $\Delta \mathrm{N}$ does not form puncta in response to ionomycin stimulation. Cells expressing mCherry-BRAG1- $\Delta \mathrm{N}$ were treated with ionomycin for 3 min and imaged as described above. $E$, Quantification of calcium-induced puncta formation. Cells expressing BRAG1-WT, BRAG1-IQ or BRAG1- $\Delta$ N were treated with either vehicle (DMSO) or ionomycin for 3 min, then fixed and imaged. Puncta were quantified as described previously (see Materials and Methods) from at least 18 cells from three independent experiments. Mean number of BRAG1 puncta per $100 \mu \mathrm{m}^{2}$ pre- and post-ionomycin stimulation (WT-pre: $4.6 \pm 0.9$ puncta, $n=19 ;$ WT-post: $66.6 \pm 13.5$ puncta; $n=19, p<0.001 ; 10$-pre: $67.5 \pm 7.4$ puncta, $n=17 ; 10$-post: $76.6 \pm 19.5$ puncta; $n=17, p=0.25 ; \Delta \mathrm{N}$-pre: $0.0 \pm 0$. puncta, $n=18 ; \Delta \mathrm{N}$-post: $13.8 \pm 3.8$ puncta; $n=19, p<0.005$ ). ${ }^{*} p \leq 0.005$ (Student's $t$ test). $\boldsymbol{F}$, The N terminus of BRAG1 is necessary for self-association. Myc-BRAG1-WT or myc-BRAG1- $\Delta$ N were coexpressed in HeLa cells with GFP-BRAG1 WT. Cell lysates were then immunoprecipitated with anti-GFP, and the presence of bound myc-BRAG1 detected by immunoblotting with anti-myc. Scale bars: $10 \mu \mathrm{m}$.
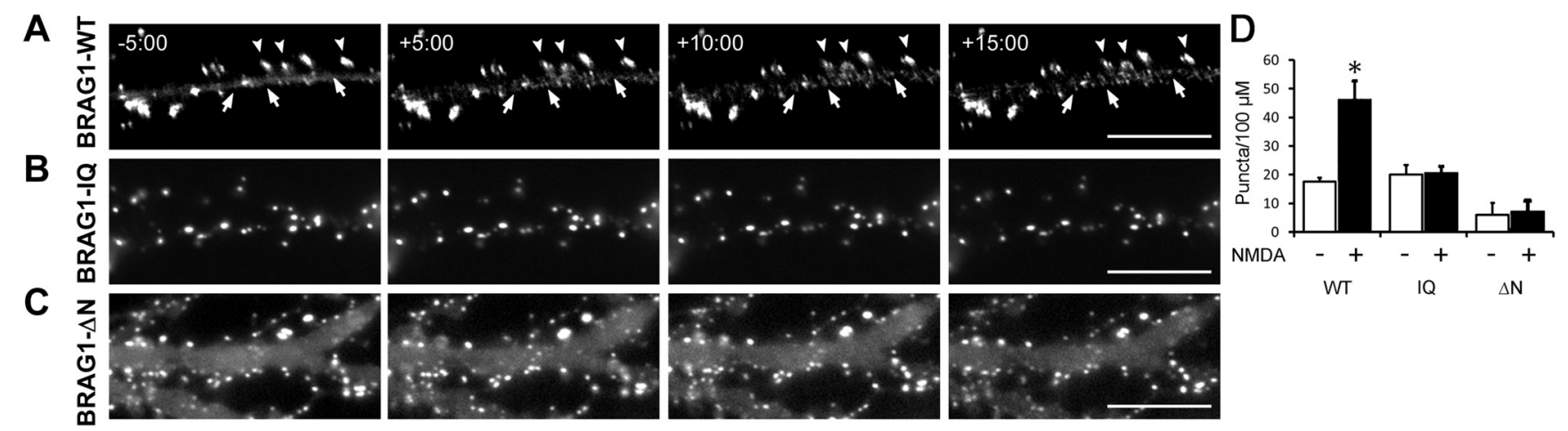

Figure 4. Stimulation of NMDA-Rs in hippocampal neurons triggers a conformational change in BRAG1. A-C, Frames from time-lapse movies of DIV 12 hippocampal neurons expressing $\mathrm{mCherry-tagged} \mathrm{BRAG1} \mathrm{constructs} \mathrm{and} \mathrm{treated} \mathrm{with} 30 \mu \mathrm{m} \mathrm{NMDA}$ for the times shown. $\boldsymbol{A}-\boldsymbol{C}$, Shows BRAG1-WT (A), BRAG1-IQ (B), and BRAG1- $\Delta \mathrm{N}(\boldsymbol{C})$. Arrowheads indicate dendritic spines where single BRAG1 foci resolve into distinct puncta. Arrows indicate formation of new puncta in the dendritic shaft. $\boldsymbol{D}$, Quantitation of puncta formation. Mean number of puncta per $100 \mu \mathrm{m}^{2}$ pre- and post-NMDA stimulation (WT-pre: $17.5 \pm 1.4$ puncta; WT-post: $46.1 \pm 6.6$ puncta; $n=3, p<0.05 ;$ IQ-pre: $20.0 \pm 3.4$ puncta; IQ-post: $20.5 \pm 2.4$ puncta; $n=4, p=0.5 ; \Delta \mathrm{N}$-pre: $6.0 \pm 4.1$ puncta; $\Delta \mathrm{N}$-post: $7.0 \pm 3.9$ puncta; $n=3, p=0.5$ ). ${ }^{*} p \leq 0.05$ (Wilcoxon). Scale bars: $10 \mu \mathrm{m}$. 
bit antiserum raised against a peptide corresponding to amino acids $258-275$ of BRAG1. As expected from previous studies, we detected endogenous BRAG1 at discrete clusters along dendrites that clearly colabel with the excitatory postsynaptic marker, PSD-95 (Fig. 2A). We next sought to confirm that exogenously expressed mCherry-tagged BRAG1 fusion proteins localized to excitatory synapses, similar to endogenous BRAG1. Therefore, we transfected dissociated rat hippocampal neurons at DIV 6 with wild-type BRAG1 fused to mCherry at its $\mathrm{N}$ terminus. Neurons were fixed at DIV 21 and counterstained for PSD-95. In contrast to soluble mCherry, which is diffusely distributed and fails to localize to any particular compartment (Fig. 2B), mCherry-BRAG1 was found in prominent puncta distributed along the length of dendrites, where it clearly colocalized with PSD-95 (Fig. 2C).

BRAG1-EK colocalized with PSD-95 to the same extent as BRAG1-WT, indicating that catalytic activity does not direct or alter BRAG1 localization. We also examined whether the IQ motif of BRAG1 was required for its localization to the PSD. Although the majority of cherry-tagged BRAG1-IQ was localized to the PSD (Fig. 2E), we detected the presence of puncta within the shaft of the dendrite that were not observed in cells expressing either BRAG1-WT or BRAG1-EK. The BRAG1- $\Delta \mathrm{N}$ mutant, which lacks the $\mathrm{N}$-terminal coiled-coil motif, also colocalizes with PSD-95 at synapses. However, we also observed a significant fraction of BRAG1- $\Delta \mathrm{N}$ diffusely distributed throughout the dendritic shaft (Fig. $2 F$ ). In summary, these results suggest that neither catalytic activity nor an intact IQ motif or coiled-coil domain is necessary for the localization of BRAG1 to the PSD.

\section{The IQ motif regulates a calcium-dependent conformational switch in BRAG1}

The calcium-dependent release of CaM from BRAG1 suggests that changes in intracellular calcium levels may regulate the BRAG1-CaM interaction, and that this might modulate BRAG1 conformation or activity. To test this idea, we examined the effects of calcium influx on mCherry-BRAG1 distribution in live HeLa cells stimulated with the calcium ionophore, ionomycin. As shown in Figure 3A, BRAG1 is mostly diffuse at steady state. However, within $30 \mathrm{~s}$ of ionomycin treatment, we observed the formation of discrete BRAG1 puncta scattered throughout the cell (Fig. 3A). These appear to be aggregates of protein, as they do not contain endosomal or other intracellular membranes (data not shown). In contrast, BRAG1-IQ exhibited a punctate distribution even in the absence of ionomycin, and did not undergo a change in its localization upon $\mathrm{Ca}^{2+}$ influx (Fig. 3B). These observations suggest that the $\mathrm{Ca}^{2+}$-induced release of CaM triggers a conformational change in BRAG1, manifested in HeLa cells as condensation into cytoplasmic puncta.

This conformational change is completely reversible, as treatment with the cell-permeable calcium chelator BAPTA-AM resulted in nearly complete dissolution of the ionomycin-induced puncta. This indicates that the redistribution of BRAG1 upon calcium influx is not simply due to protein degradation or denaturation, and likely involves a regulated change in BRAG1 conformation. Quantitation of this phenomenon indicated an $\sim 15$-fold increase in the number of BRAG1-WT puncta after ionomycin treatment, which was statistically indistinguishable from BRAG1-IQ in the absence of ionomycin (Fig. 3D).

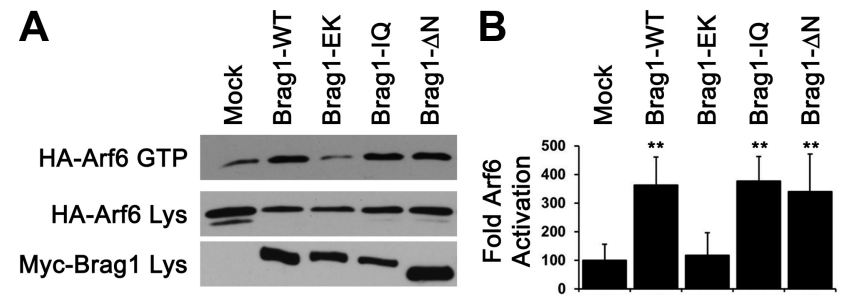

Figure 5. The IQ motif and $\mathrm{N}$ terminus are dispensable for BRAG1 catalytic activity in heterologous cells. A, Pulldown assay for Arf6 activity. Active GTP-bound Arf6 was precipitated with GST-GGA3 from lysates of HeLa cell coexpressing Arf6-HA and the indicated myc-tagged BRAG1 constructs. $B$, Quantitation of Arf6 activation (Ctrl: $100.0 \pm 55.6 \%$; WT: $362.0 \pm 99.4 \% ; n=3$; $p<0.05$; EK: $117.3 \pm 78.9 \% ; n=3 ; p=0.5 ;$ IQ: $376.8 \pm 86.0 \% ; n=3 ; p<0.05 ; \Delta \mathrm{N}$ $340.6 \pm 131.3 \% ; n=3 ; p<0.05$ ). The relative values and SEs were normalized to average amounts of Arf6-GTP from control cells expressing Arf6-HA alone. ${ }^{* *} p<0.05$ (Wilcoxon test).

\section{The $\mathrm{N}$ terminus of BRAG1 mediates calcium-dependent self-association}

Because coiled-coil domains commonly mediate homooligomerization or protein-protein interactions (Burkhard et al., 2001), we speculated that the N-terminal BRAG1 coiled-coil domain (residues 23-74) plays a role in its calcium-induced selfassociation. Deletion of this domain (BRAG1- $\Delta \mathrm{N})$ did not affect the steady-state distribution of BRAG1 in HeLa cells (Fig. 3E). However, unlike wild-type BRAG1, BRAG1- $\Delta$ N remained diffusely cytosolic upon addition of ionomycin (Fig. $3 E$ ). This observation indicates that $\mathrm{Ca}^{2+}$-induced self-association of wild-type BRAG1 is dependent upon the $\mathrm{N}$-terminal coiledcoil domain.

To support this hypothesis, we tested the ability of BRAG1 to oligomerize. For this purpose, GFP-tagged BRAG1-WT was expressed in HeLa cells along with either myc-tagged BRAG1-WT or myc-BRAG1- $\Delta \mathrm{N}$. When GFP-BRAG1-WT was immunoprecipitated with anti-GFP antibody, we found that myc-BRAG1-WT coprecipitated efficiently while myc-BRAG1- $\Delta \mathrm{N}$ did not (Fig. $3 F$ ). This observation indicates that BRAG1 can oligomerize via its $\mathrm{N}$-terminal coiled-coil domain, and suggests that regulated oligomerization, induced by CaM release, may have an important role in BRAG1 function in the synapse.

An influx of extracellular calcium is known to occur upon activation of NMDA-Rs. To determine whether BRAG1 responds to physiological levels of calcium in the neuronal context, we expressed mCherry-tagged BRAG1-WT in cultured hippocampal neurons and followed its localization after NMDA stimulation using live cell imaging (Fig. 4A). Before stimulation, BRAG1-WT was stably localized to the PSD. However, after the addition of $30 \mu \mathrm{M}$ NMDA, small BRAG1 puncta appeared within spines and within the dendritic shaft, in addition to its normal synaptic localization. These smaller puncta were reminiscent of those seen in HeLa cells after ionomycin stimulation, and are consistent with the idea of calcium-induced self-association of BRAG1. We also examined the effects of NMDA stimulation on the distribution of BRAG1-IQ and BRAG1- $\Delta \mathrm{N}$ in hippocampal neurons (Fig. $4 B, C$ ). Similar to our findings in HeLa cells treated with ionomycin, we saw no detectable changes in the distribution of either mutant after NMDA stimulation (Fig. 4D). This suggested that the NMDA-induced condensation of BRAG1 in hippocampal neurons requires both the IQ and the coiled-coil motifs.

CaM binding is not required for BRAG1 catalytic activity To test whether the IQ domain or the $\mathrm{N}$ terminal coiled-coil domain regulates BRAG1 Arf-GEF activity, we measured their 


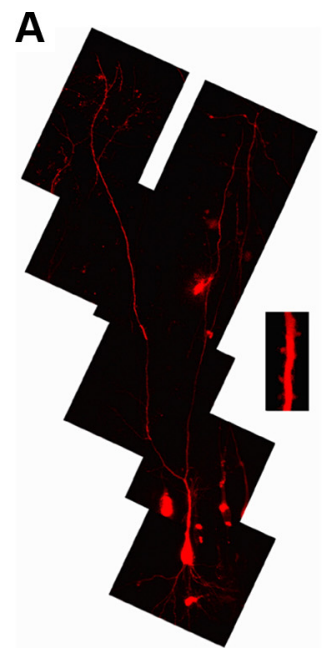

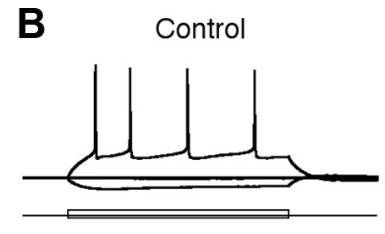

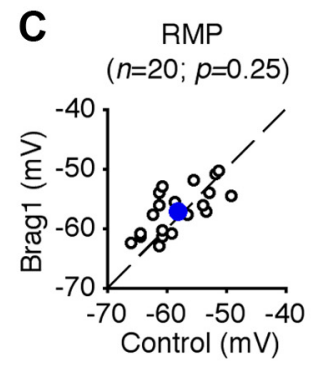

BRAG1

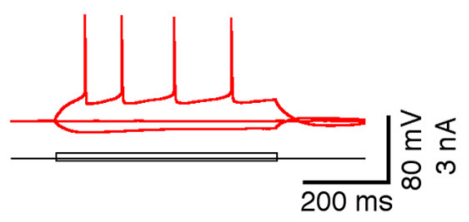

$\mathrm{R}_{\text {input }}$ $(n=20 ; p=0.50)$

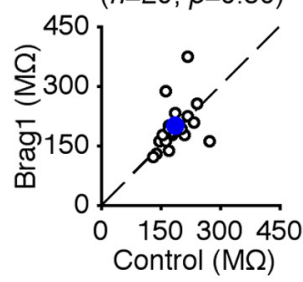

Time Constant $(n=20 ; p=0.97)$

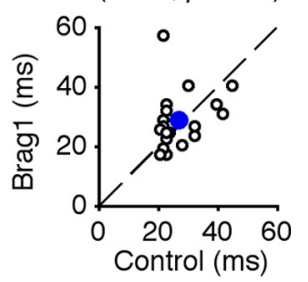

Figure 6. Expression of BRAG1 does not alter the basic membrane properties of CA1 neurons. $A$, Two-photon fluorescence imaging indicates that mCherry-BRAG1 is expressed in the dendrites and spines (inset) of CA1 pyramidal neurons. B, Evoked responses to step depolarizing and hyperpolarizing pulses recorded from neighboring nonexpressing (Ctrl) and BRAG1-expressing cells. C, Resting membrane potentials (Ctrl: $-58.1 \pm 1.1 \mathrm{mV} ; \mathrm{BRAG1}:-57.2 \pm 0.9 \mathrm{mV} ; n=20 ; p=0.25$ ), input resistances (Ctrl: 189.8 \pm 8.2 M $\Omega ;$ BRAG1: 190.2 $\pm 13.2 \mathrm{M} \Omega ; n=20 ; p=0.50)$, and time constants (Ctrl:27.3 \pm 1.7 ms; BRAG1: $28.5 \pm 2.1 \mathrm{~ms} ; n=20 ; p=0.97)$ in mCherry-BRAG1-expressing cells are plotted against those obtained from control nonexpressing cells. Blue dots indicate the average points.

A

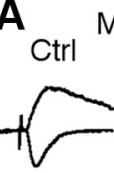

+ APV
Media

BRAG1-WT<smiles>CCCCCOCCOCCOCCO</smiles>

Ctr

Media

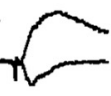

BRAG1-AN

Ctrl
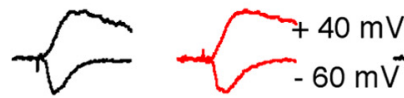

Ctr

$+\mathrm{APV}$
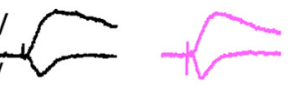

B $\square$ Ctrl $\square$ BRAG1-WT BRAG1- $\triangle N$
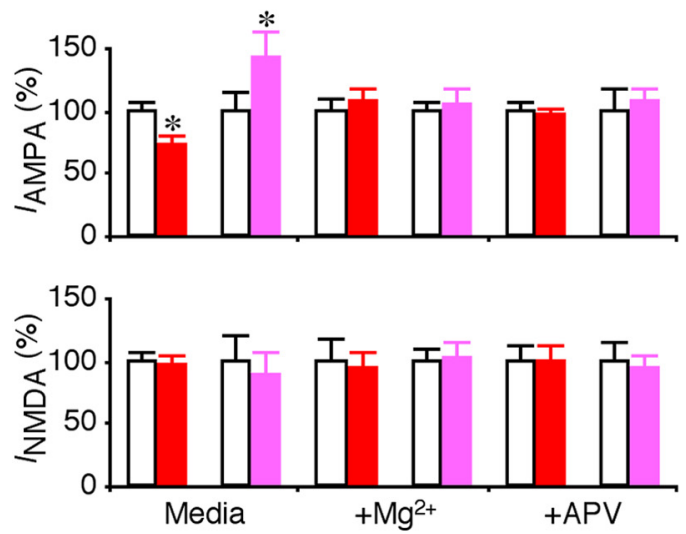

C

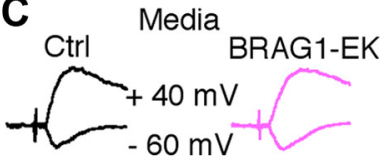

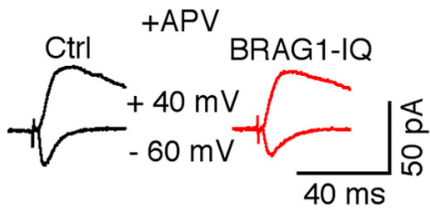

D DCtrl BRAG1-EK \#BRAG1-IQ
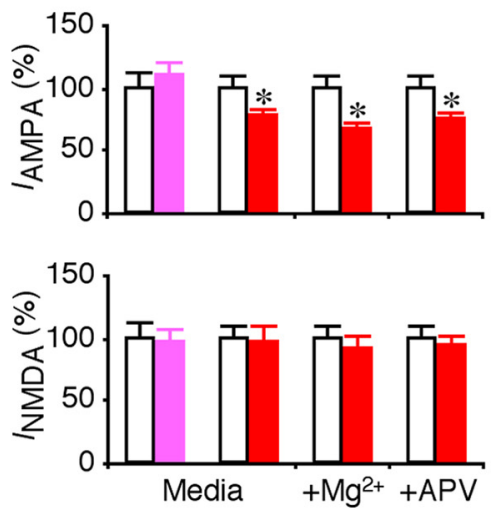

Figure 7. BRAG1-WT and BRAG1 mutants differentially affect synaptic transmission. $A$, Evoked AMPA-R-mediated $(-60 \mathrm{mV})$ and NMDA-R-mediated $(+40 \mathrm{mV})$ responses recorded from neighboring nonexpressing (Ctrl) and mCherry-tagged BRAG1-WT- or BRAG1- $\Delta \mathrm{N}$-expressing neurons cultured in normal media or media containing $100 \mu \mathrm{m} \mathrm{DL}-A$ PV. $\boldsymbol{B}$, Values for AMPA responses in neurons expressing BRAG1-WT and BRAG1- $\Delta$ N cultured in normal media (Ctrl: $-31.6 \pm 2.5 \mathrm{pA} ; \mathrm{WT}:-22.9 \pm 2.4 \mathrm{pA} ; n=38 ; p<0.05 ;$;trl: $-14.2 \pm 2.1 \mathrm{pA} ; \Delta \mathrm{N}:-20.1 \pm 3.2 \mathrm{pA} ; n=16 ; p<0.05)$, in media containing high $\mathrm{Mg}^{2+}$ (Ctrl: $\left.-26.8 \pm 2.6 \mathrm{pA} ; \mathrm{WT}:-28.8 \pm 3.1 \mathrm{pA} ; n=22 ; p=0.94 ; \mathrm{Ctrl}:-20.3 \pm 1.7 \mathrm{pA} ; \Delta \mathrm{N}:-21.4 \pm 2.3 \mathrm{pA} ; n=23 ; p=0.93\right)$, or in media containing DL-APV (Ctrl: $-31.7 \pm 2.3 \mathrm{pA} ; \mathrm{WT}$ : $-30.1 \pm 2.4 \mathrm{pA} ; n=19 ; p=0.51 ;$ (trl: $-22.3 \pm 3.8 \mathrm{pA} ; \Delta \mathrm{N}:-23.8 \pm 2.5 \mathrm{pA} ; n=19 ; p=0.69$ ), and for NMDA responses in neurons expressing Brag1-WT and BRAG1- $\Delta \mathrm{N}$ cultured in normal media (Ctrl: $79.3 \pm 6.5 \mathrm{pA} ; \mathrm{WT}: 75.9 \pm 6.8 \mathrm{pA} ; n=38 ; p=0.60 ;(\operatorname{trl}: 46.6 \pm 9.7 \mathrm{pA} ; \Delta \mathrm{N}: 41.3 \pm 8.5 \mathrm{pA} ; n=16 ; p=0.33)$, in media containing high Mg${ }^{2+}(\mathrm{Ctrl}: 66.4 \pm 11.6 \mathrm{pA} ; \mathrm{WT}: 61.6 \pm 9.4 \mathrm{pA} ; n=22 ; p=0.78 ;$ Ctrl: $53.8 \pm 5.3 \mathrm{pA} ; \Delta \mathrm{N}: 54.3 \pm 7.4 \mathrm{pA} ; n=23 ; p=0.72$ ), or in media containing DL-APV (Ctrl:59.2 $\pm 7.3 \mathrm{pA} ; \mathrm{WT}: 59.3 \pm 6.7 \mathrm{pA} ; n=19 ; p=0.94 ;$ (trl: $47.4 \pm 6.8 \mathrm{pA} ; \Delta \mathrm{N}: 44.4 \pm 5.5 \mathrm{pA} ; n=19 ; p=$ 0.57 ) related to control nonexpressing neurons. C, Evoked AMPA-R-mediated ( $-60 \mathrm{mV}$ ) and NMDA-R-mediated ( $+40 \mathrm{mV}$ ) responses recorded from neighboring nonexpressing (Ctrl) with either mCherrytagged BRAG1-EK or BRAG1-IQ expressing neurons cultured in normal media or media containing $100 \mu \mathrm{MDL}-A$ PV. D, Valuesfor AMPA responses in neurons expressing BRAG1-EK and BRAG1-IQ cultured in normal media (Ctrl: $-24.1 \pm 2.8 \mathrm{pA} ; \mathrm{EK}:-26.5 \pm 2.5 \mathrm{pA} ; n=21 ; p=0.52 ;$ Ctrl: $-25.4 \pm 2.6 \mathrm{pA} ; \mathrm{lQ}:-19.4 \pm 1.9 \mathrm{pA} ; n=25 ; p<0.05)$, in neurons expressing BRAG1-IQ cultured in media containing high Mg ${ }^{2+}$ (Ctrl: $-23.0 \pm 2.3 \mathrm{pA} ; \mathrm{IQ}:-15.4 \pm 1.3 \mathrm{pA} ; n=25 ; p<0.005$ ), or DL-APV (Ctrl: $-26.7 \pm 2.8 \mathrm{pA} ; \mathrm{IQ}:-19.9 \pm 1.7 \mathrm{pA} ; n=24 ; p<0.01$ ), and for NMDA responses in neurons expressing BRAG1-EK or BRAG1-IQ cultured in normal media (Ctrl: $77.1 \pm 9.1 \mathrm{pA} ; \mathrm{EK}: 74.1 \pm 8.0 \mathrm{pA} ; n=21 ; p=0.54 ;$ Ctrl: $52.5 \pm 5.2 \mathrm{pA} ; \mathrm{IQ}: 50.8 \pm 6.6 \mathrm{pA} ; n=25 ; p=0.14)$, in neurons expressing BRAG1-IQ cultured in media containing high $\mathrm{Mg}^{2+}(\mathrm{Ctrl}: 57.6 \pm 5.7 \mathrm{pA} ; \mathrm{lQ}: 52.9 \pm 5.7 \mathrm{pA} ; n=25 ; p=0.20)$, or DL-APV (Ctrl: $\left.68.9 \pm 6.8 \mathrm{pA} ; \mathrm{lQ}: 64.4 \pm 5.8 \mathrm{pA} ; n=24 ; p=0.67\right)$ related to control nonexpressing neurons. The relative values and SEs were normalized to average amounts of AMPA and NMDA responses from control CA1 cells. ${ }^{*} p<0.05$ (Wilcoxon test). 
ability to activate Arf6 in HeLa cells using a previously described GST-GGA3 pulldown assay (Santy and Casanova, 2001) to specifically precipitate GTPbound Arf6. Coexpression of BRAG1-WT with Arf6 in HeLa cells enhanced Arf6 activation fourfold relative to cells expressing Arf6 alone (Fig. 5A,B). As expected, the catalytically inactive mutant BRAG1E849K failed to activate Arf6 above basal levels. Surprisingly, both BRAG1-IQ and BRAG1- $\Delta$ N mutants significantly stimulated Arf6 activity, although the BRAG1$\Delta \mathrm{N}$-mediated activity was slightly lower than BRAG1-WT.

\section{Activation of BRAG1 depresses AMPA- $\mathrm{R}$-mediated transmission in \\ CA1 neurons}

To further examine the synaptic functions of BRAG1, we used recombinant Sindbis virus to acutely overexpress mCherry-BRAG1 in CA1 pyramidal neurons of rat hippocampal cultured slices. In expressing neurons, mCherry-BRAG1 was diffusely distributed and infiltrated in dendritic spines, the sites of excitatory synapses (Fig. 6A). Electrophysiological recordings were obtained simultaneously from nearby expressing and nonexpressing neurons. We found that expression of mCherry-BRAG1 had no effects on basic membrane properties, including resting membrane potentials, input resistance, and membrane time constants (Fig. $6 B, C$ ). We then examined EPSCs in expressing neurons and nearby control nonexpressing neurons by stimulating the afferent fibers. Neurons expressing wild-type BRAG1 exhibited depressed (by $~ 30 \%$ ) AMPA-R-mediated responses compared with nearby nonexpressing controls (Fig. $7 A, B$ ), suggesting that activating BRAG1 depresses transmission. Interestingly, expression of BRAG1- $\Delta \mathrm{N}$ did not suppress AMPA-R activity, but instead potentiated it (Fig. $7 A, B)$, suggesting a possible dominant-negative effect. No significant difference was observed in NMDA-R-mediated responses between BRAG1-expressing and nonexpressing neurons (Fig. 7A,B), suggesting a postsynaptic mechanism.

To determine whether BRAG1 signaling is stimulated by synaptic and NMDA-R activity, we included $12 \mathrm{mM} \mathrm{MgCl}_{2}$, which depresses synaptic transmission (Zhu et al., 2000), or DL-APV, a pharmacological blocker of NMDA-Rs, in culture media during expression of BRAG1. Both high $\mathrm{Mg}^{2+}$ and APV completely blocked the effects of both BRAG1-WT and BRAG1- $\Delta$ N expression on AMPA synaptic transmission (Fig. $7 A, B$ ). These results indicate that spontaneous synaptic activity activates NMDA-Rs that in turn activate BRAG1, producing a tonic depression of AMPA-R-mediated transmission.

To examine how mutations in the catalytic or IQ domains might affect synaptic transmission, we expressed mCherrytagged BRAG1-EK or BRAG1-IQ in CA1 neurons. In contrast to wild-type BRAG1, which depressed AMPA responses, neurons expressing the catalytically inactive BRAG1-EK mutant responded similarly to controls, indicating that BRAG1 catalytic activity is necessary for the observed depression observed upon expression of the wild-type protein (Fig. $7 C, D$ ). The IQ domain mutant reduced AMPA responses to a similar extent as the wildtype protein, consistent with its retention of catalytic activity (Fig. 7C,D). However unlike BRAG1-WT, which is entirely dependent upon NMDA-R signaling, the depressive effect of BRAG1-IQ was
B 口Ctrl $\operatorname{BRAG1-IQ}$
Arf6-GTP

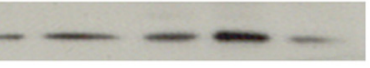

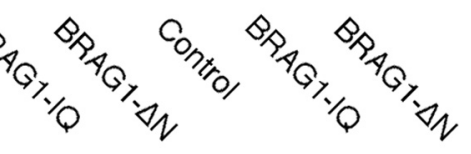

BRAG1- $\triangle N$

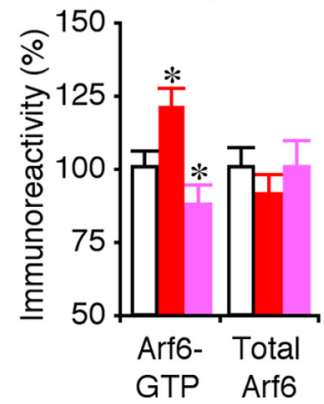

Figure 8. BRAG1 regulates endogenous Arf6 signaling. A, Relative levels of GTP-bound Arf6 and total Arf6 in control hippocam-

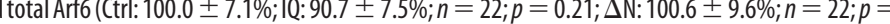
86) in control hippocampal CA1 regions, and hippocampal CA1 regions expressing BRAG1-IQ or BRAG1- $\Delta \mathrm{N}$. The relative values ge amounts of Arf6-GTP or total Arf6 from control untreated cells. * $p<0.05$ (Wilcoxon test)

not blocked by high $\mathrm{Mg}^{2+}$ or APV. This observation suggests that the inability to interact with CaM abrogates the requirement for NMDA-R activation, and renders this mutant constitutively active.

\section{BRAG1-Arf6 stimulates JNK-mediated synaptic GluA1 removal in CA1 neurons}

To determine how BRAG1 depresses synaptic transmission, we examined whether BRAG1 mutants affect endogenous Arf6 signaling in CA1 neurons in hippocampal cultured slices using the GST-GGA3 pulldown assay (Santy and Casanova, 2001). As shown in Figure 8, CA1 cells expressing BRAG1-IQ displayed increased levels of active Arf6-GTP, whereas those expressing BRAG1- $\Delta \mathrm{N}$ had decreased levels of active Arf6-GTP compared with control nonexpressing CA1 cells, indicating that BRAG1-IQ stimulates and BRAG1- $\Delta \mathrm{N}$ inhibits endogenous Arf6 activity in neurons.

We then investigated whether BRAG1-Arf6 signals synaptic depression by stimulating the JNK and p38 MAPK signaling pathways, which depress transmission by stimulating synaptic removal of AMPA-Rs (Zhu et al., 2002, 2005; Kielland et al., 2009). To test this idea, we included SP600125, an inhibitor of JNK (Bennett et al., 2001), or SB203580, which inhibits p38 MAPK (Lee et al., 1999), in culture media during expression of BRAG1-IQ and BRAG1- $\Delta$ N. SP600125, but not SB203580, completely blocked the depressive effect of BRAG1-IQ and the potentiative effect of BRAG1- $\Delta \mathrm{N}$ in CA1 neurons (Fig. 9A,B), suggesting a selective involvement of JNK signaling. Consistent with this idea, Western blots showed that expression of BRAG1-IQ increased levels of phosphorylated JNK in CA1 cells, while expression of BRAG1- $\Delta \mathrm{N}$ decreased JNK activation (Fig. $9 C, D)$. Importantly, neither construct affected the levels of p38 MAPK phosphorylation (Fig. 9C,D). Expression of BRAG1-IQ or BRAG1 $-\Delta \mathrm{N}$ did not alter the levels of total JNK and p38 (Fig. $9 C, D)$. Collectively, these results indicate that BRAG1-Arf6 signals synaptic depression via stimulating JNK signaling, but not p38 MAPK signaling.

JNK and p38MAPK depress transmission by signaling synaptic removal of GluA1- and GluA2-containing AMPA-Rs, respectively (Zhu et al., 2002, 2005; Kielland et al., 2009). To test whether BRAG1-Arf6 regulates synaptic trafficking of GluA1and/or GluA2-containg AMPA-Rs, we examined the effects of 

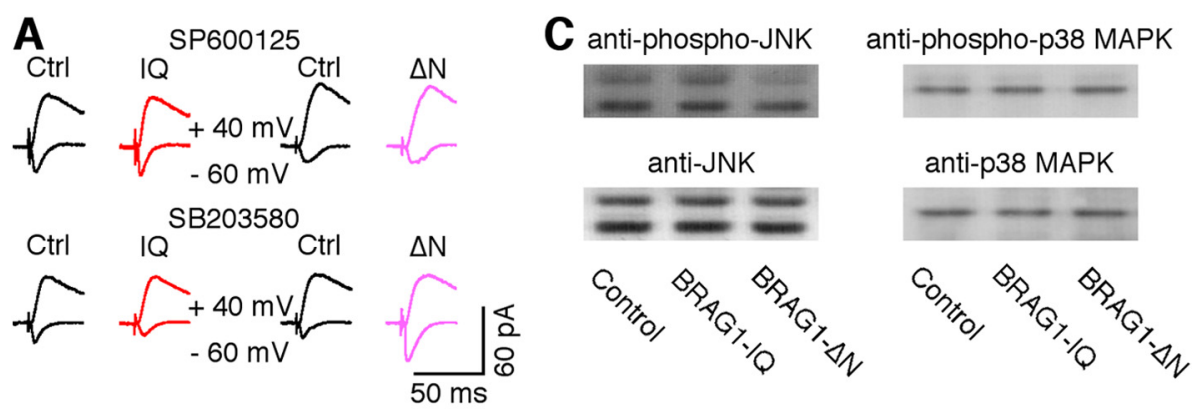

\section{B $\square$ Ctrl $\square$ BRAG1-IQ $\square$ BRAG1- $\Delta N$}
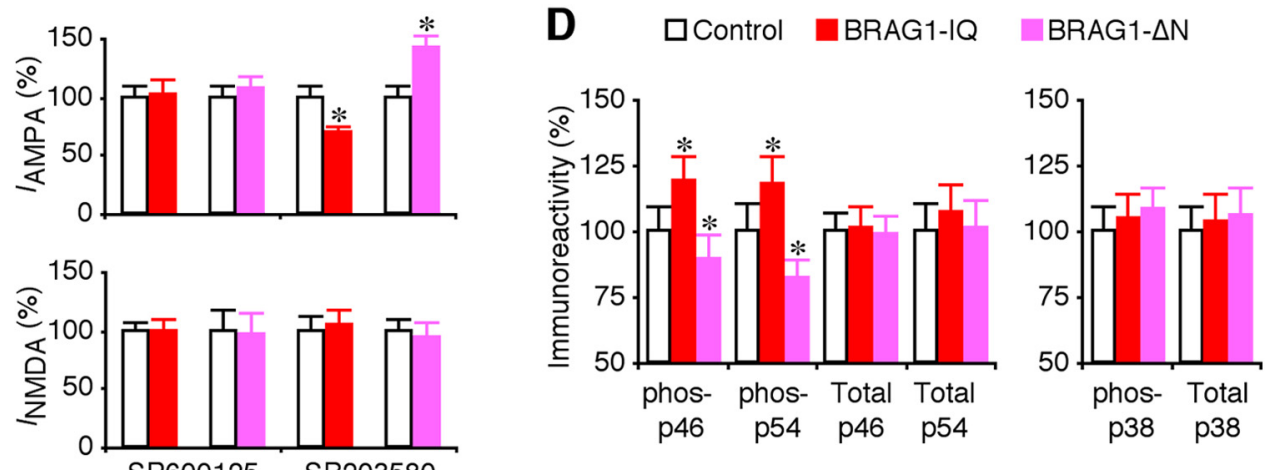

Figure 9. BRAG1 signals synaptic depression via JNK. A, Evoked AMPA-R-mediated ( $-60 \mathrm{mV}$ ) and NMDA-R-mediated $(+40 \mathrm{mV})$ responses recorded from neighboring nonexpressing (Ctrl) and

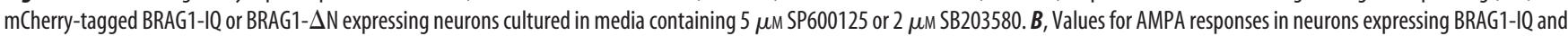
BRAG1- $\Delta \mathrm{N}$ cultured in media containing SP600125 (Ctrl: $-23.0 \pm 2.1 \mathrm{pA} ; \mathrm{IQ}:$ minus] $23.5 \pm 2.8 \mathrm{pA} ; n=26, p=0.81 ;$ (trl: $-18.4 \pm 1.6 \mathrm{pA} ; \Delta \mathrm{N}:-19.7 \pm 2.0 \mathrm{pA} ; n=23, p=0.48$ ) or SB203580 (Ctrl: $-26.0 \pm 2.2 \mathrm{pA} ; \mathrm{lQ}:-18.2 \pm 1.4 \mathrm{pA} ; n=28, p<0.005 ;$ (trl: $-17.9 \pm 1.6 \mathrm{pA} ; \Delta \mathrm{N}:-25.5 \pm 1.9 \mathrm{pA} ; n=23, p<0.01$ ), and for NMDA responses in neurons expressing BRAG1-IQ and BRAG1- $\Delta \mathrm{N}$ cultured in media containing SP600125 (Ctrl:55.4 $\pm 4.7 \mathrm{pA} ; \mathrm{lQ}: 55.4 \pm 6.0 \mathrm{pA} ; n=26, p=0.53 ;$ (trl: $60.7 \pm 10.6 \mathrm{pA} ; \Delta \mathrm{N}: 58.3 \pm 12.1 \mathrm{pA} ; n=23, p=0.38)$ or SB203580 (Ctrl:51.3 \pm $6.7 \mathrm{pA} ; \mathrm{lQ}: 54.0 \pm 6.2 \mathrm{pA} ; n=28, p=0.93 ;$ (trll: $60.6 \pm 6.3 \mathrm{pA} ; \mathrm{IQ}: 56.4 \pm 7.7 \mathrm{pA} ; n=23, p=0.43$ ) related to control nonexpressing neurons. C, Western blots of phospho-p46/54 JNK or p38 MAPK in control hippocampal CA1 regions, hippocampal CA1 regions expressing BRAG1-IQ, and hippocampal CA1 regions expressing BRAG1- $\Delta \mathrm{N}$. Each lane was loaded with the same amount of protein (45 $\mu \mathrm{g}$ ). D, Left, Relative amounts of phospho-p46 (Ctrl: $100.0 \pm 5.3 \% ; 10: 118.9 \pm 7.5 \% ; n=12 ; p<0.005 ; \Delta \mathrm{N}: 89.6 \pm 5.8 \% ; n=12 ; p<0.01$ ) and phospho-p54 (Ctrl: 100.0 $\pm 11.0 \%$; IQ: $117.4 \pm 11.6 \% ; n=12 ; p<0.005 ; \Delta \mathrm{N}: 81.6 \pm 9.9 \% ; n=12 ; p<0.005)$ JNK in hippocampal CA1 regions expressing BRAG1-IQ and BRAG1- $\Delta \mathrm{N}$. Relative amounts of total p46 (Ctrl: $100.0 \pm$ 7.7\%; IQ: $101.2 \pm 8.2 \% ; n=12 ; p=0.64 ; \Delta \mathrm{N}: 98.3 \pm 8.1 \% ; n=12 ; p=0.43)$ and total $\mathrm{p} 54(\mathrm{Ctrl}: 100.0 \pm 10.2 \% ; \mathrm{lQ}: 107.2 \pm 11.0 \% ; n=12 ; p=0.14 ; \Delta \mathrm{N}: 101.3 \pm 10.2 \% ; n=12 ; p=$ 0.24) JNK in hippocampal CA1 regions expressing BRAG1-IQ and BRAG1- $\Delta$ N. Right, Relative amounts of phospho-p38 MAPK (Ctrl: 100.0 $\pm 9.1 \% ; 1 \mathrm{IQ}: 105.1 \pm 9.5 \% ; n=12 ; p=0.31 ; \Delta \mathrm{N}: 107.9 \pm$ $8.7 \% ; n=12 ; p=0.11$ ) in hippocampal CA1 regions expressing BRAG1-IQ and BRAG1- $\Delta \mathrm{N}$. Relative amounts of total p38 MAPK (Ctrl: $100.0 \pm 9.9 \% ; 10: 103.0 \pm 10.8 \% ; n=12 ; p=0.64 ; \Delta \mathrm{N}$ : $106.0 \pm 10.7 \% ; n=12 ; p=0.94$ ) in hippocampal CA1 regions expressing BRAG1-IQ and BRAG1- $\Delta \mathrm{N}$. The relative values and SEs were normalized to average amounts of AMPA and NMDA responses from control CA1 cells, phospho-JNK, phospho-p38 MAPK, total JNK, or total p38 MAPK from control hippocampal CA1 regions. * $p<0.05$ (Wilcoxon test).

BRAG1 mutants in CA1 neurons prepared from GluA1 and GluA2 knock-out mice. As shown in Figure 10, the depressive effect of BRAG1-IQ and potentiative effect of BRAG1- $\Delta$ N were occluded or blocked in GluA1 but not GluA2 knock-out CA1 neurons. These results suggest that BRAG1-Arf6 signals synaptic depression via stimulating JNK-mediated synaptic removal of GluA1-containing AMPA-Rs.

\section{Discussion}

The Arf GEFs BRAG1, BRAG2, and BRAG3 are highly enriched in the brain, where they are concentrated in postsynaptic densities. While all three BRAG family proteins are expressed in hippocampal neurons, BRAG3 localizes specifically to the PSDs of inhibitory synapses, whereas both BRAG1 and BRAG2 are found at excitatory synapses (Sakagami et al., 2008; Scholz et al., 2010; Fukaya et al., 2011). While BRAG2 was recently shown to regulate mGluR-dependent synaptic removal of GluA2-containing AMPA-Rs (Scholz et al., 2010), the synaptic function of BRAG1, which is implicated in nonsyndromic XLID (Shoubridge et al., 2010), had not been investigated. Here we report that BRAG1 signals synaptic depression of AMPA transmission in response to synaptic activation of NMDA-Rs. We further show that diseaseassociated mutations, which affect either catalytic activity or CaM binding, result in either inhibition or constitutive activation of Arf6 signaling, respectively. Moreover, while BRAG2 acts on GluA2-containing AMPA-Rs (Scholz et al., 2010), BRAG1 appears to selectively modulate GluA1-containing AMPA-Rmediated transmission through a mechanism that involves the downstream activation of JNK. These observations provide new insight into the machinery controlling AMPA-R trafficking, and provide a mechanistic basis for the defects in learning and memory exhibited by patients with XLID.

\section{Mechanisms of BRAG1-Arf6 signaling}

Synaptic NMDA-R activation induces a rapid local increase in $\mathrm{Ca}^{2+}$ levels that is critical for the induction of synaptic plasticity (Malenka et al., 1988). The IQ motif is evolutionarily conserved among the BRAG family Arf-GEFs, and although it has been assumed to bind CaM, this had not been previously demonstrated. Here we provide the first evidence that the BRAG1 IQ motif does indeed bind CaM, and that it preferentially interacts with the $\mathrm{Ca}^{2+}$-free form. We also show that CaM dissociation triggered by $\mathrm{Ca}^{2+}$ influx induces a conformational change in BRAG1 resulting in a change in subcellular distribution. However, while CaM binding clearly impacts conformation, its relationship to BRAG1 function is complex. In heterologous cells, 
A<smiles>CCC(C)C</smiles>

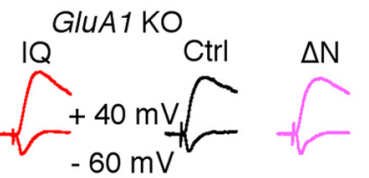

Ctrl<smiles></smiles>

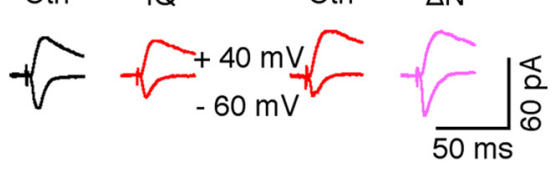

\section{B $\square$ Ctrl $\square$ BRAG1-IQ $\square$ BRAG1- $\triangle \mathrm{N}$}
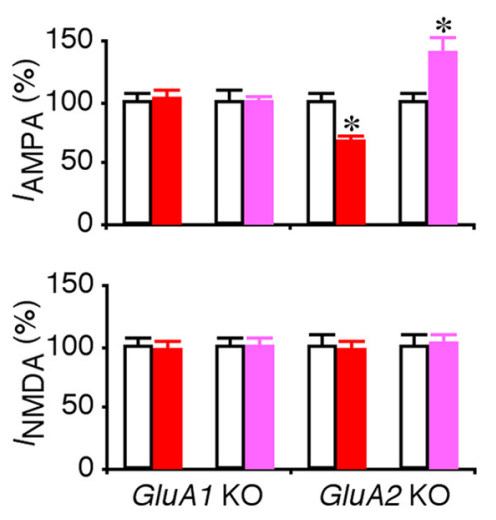

Figure 10. BRAG1 signaling regulates synaptic trafficking of GluA1-containing AMPA-Rs. A, Evoked AMPA-R-mediated $(-60 \mathrm{mV})$ and NMDA-R-mediated $(+40 \mathrm{mV})$ responses recorded from neighboring nonexpressing (Ctrl) and mCherry-tagged BRAG1-IQ or BRAG1- $\Delta$ N expressing neurons prepared from GluA1 or GluA2 knock-out mice. $\boldsymbol{B}$, Values for AMPA responses in BRAG1-IQ and BRAG1- $\Delta$ N expressing neurons prepared from GluA1 (Ctrl: $-23.5 \pm 1.8 \mathrm{pA}$; IQ: $-24.1 \pm 2.0 \mathrm{pA} ; n=20, p=0.88 ; \mathrm{Ctrl}:-26.2 \pm 2.5 \mathrm{pA} ; \Delta \mathrm{N}:-26.2 \pm 1.5 \mathrm{pA} ; n=20, p=$ 0.82 ) or GluA2 (Ctrl: $-30.9 \pm 2.1 \mathrm{pA} ; \mathrm{IQ}:-20.9 \pm 1.6 \mathrm{pA} ; n=20, p<0.005 ;$ Ctrl: $-24.5 \pm$ $1.7 \mathrm{pA} ; \Delta \mathrm{N}:-31.5 \pm 2.9 \mathrm{pA} ; n=24, p<0.005)$ knock-out mice, and for NMDA responses in BRAG1-IQ and BRAG1- $\Delta$ N expressing neurons prepared from GluA1 (Ctrl: $94.9 \pm 7.6$ pA; IQ: $91.9 \pm 8.4 \mathrm{pA} ; n=20, p=0.91 ;(\mathrm{trl}: 113.6 \pm 8.7 \mathrm{pA} ; \Delta \mathrm{N}: 111.9 \pm 9.8 \mathrm{pA} ; n=20, p=0.97)$ or GluA2 (Ctrl: $54.5 \pm 5.0 \mathrm{pA} ; \mathrm{IQ}: 54.8 \pm 5.2 \mathrm{pA} ; n=20, p=0.46 ;$ (trl: $60.1 \pm 5.2 \mathrm{pA} ; \Delta \mathrm{N}$ : $61.8 \pm 4.4 \mathrm{pA} ; n=24, p=0.63)$ knock-out mice. The relative values and SEs were normalized to average amounts of AMPA and NMDA responses from control CA1 cells. ${ }^{*} p<0.05$ (Wilcoxon test).

BRAG1 catalytic activity appears to be constitutive and is not affected by mutations in the IQ motif that abrogate CaM binding. Similarly, disruption of the catalytic domain, but not the IQ motif, of the single Drosophila BRAG gene Loner was found to cause defects in myoblast fusion (Chen et al., 2003). However, our results show that in hippocampal neurons BRAG1 activity is tightly regulated, requiring upstream NMDA-R activity. $\mathrm{Mu}$ tation of the IQ motif relieves this constraint, allowing AMPA-R downregulation in the absence of NMDA-R activity. These observations suggest a model in which NMDA-R-mediated $\mathrm{Ca}^{2+}$ influx triggers the release of CaM from BRAG1, which then stimulates AMPA-R endocytosis via its activation of Arf6 (Fig. 11). They also provide a mechanistic explanation for how mutation of the IQ motif found in one family with XLID may cause disease: failure to bind CaM leads to constitutive BRAG1 activity, resulting in chronic downregulation of AMPA-R signaling.

The responsiveness of BRAG1 to $\mathrm{Ca}^{2+}$ in the neuronal context is presumably due to the presence of neuron-specific binding partners that help anchor it in the PSD or mediate interactions with other proteins involved in AMPA-R trafficking. In this regard it is interesting that a BRAG1 mutant lacking the N-terminal coiled-coil domain actually potentiates AMPA responses, suggesting that it acts as a dominant-negative to inhibit the function of endogenous BRAG1. This hypothesis is supported by the observation that both endogenous Arf6 activity and JNK activity are reduced in the presence of BRAG1- $\Delta \mathrm{N}$. Because BRAG1- $\Delta \mathrm{N}$ is more diffusely distributed within the dendritic shaft and spines, it may bind and sequester components that are limiting for receptor internalization, JNK activation, or both.

\section{Signaling interaction of BRAG1-Arf6 and Rap2-JNK-PP2B}

In this study, we provide the first evidence that BRAG1-Arf6 signaling intersects the Rap2-MINK-JNK-PP2B signaling pathway at synapses. Previous studies have shown that synaptic activation of NMDA-Rs increases Rap2 signaling, which controls dephosphorylation and synaptic removal of GluA1-containing AMPA-Rs during depotentiation via stimulating the MINKJNK-PP2B signaling pathway (Zhu et al., 2005; Kielland et al., 2009; Hussain et al., 2010; Yang et al., 2011). We show here that synaptic activity also stimulates BRAG1-Arf6 activity. Interestingly, activation of BRAG1-Arf6 depresses synaptic transmission via stimulating JNK, and blocking JNK activity blocks BRAG1Arf6-mediated synaptic depression. These results are consistent with previous observations that Arf6 can signal downstream via a neuronal scaffolding protein JIP3 (Montagnac et al., 2009; Suzuki et al., 2010), and that JIP3 regulates JNK signaling(Kelkar et al., 2000; Ongusaha et al., 2008). Moreover, the BRAG1-mediated synaptic depression, which requires Arf6 activation, is mediated by synaptic trafficking of GluA1-containing AMPA-Rs. Together, these results suggest that BRAG1-Arf6 depresses synaptic transmission via regulating Rap2-JNK-PP2B signaling.

\section{Altered BRAG1 signaling in X-linked mental disability}

Our results suggest a novel synaptic signaling mechanism whose dysregulation results in X-linked mental retardation. Previous stud-

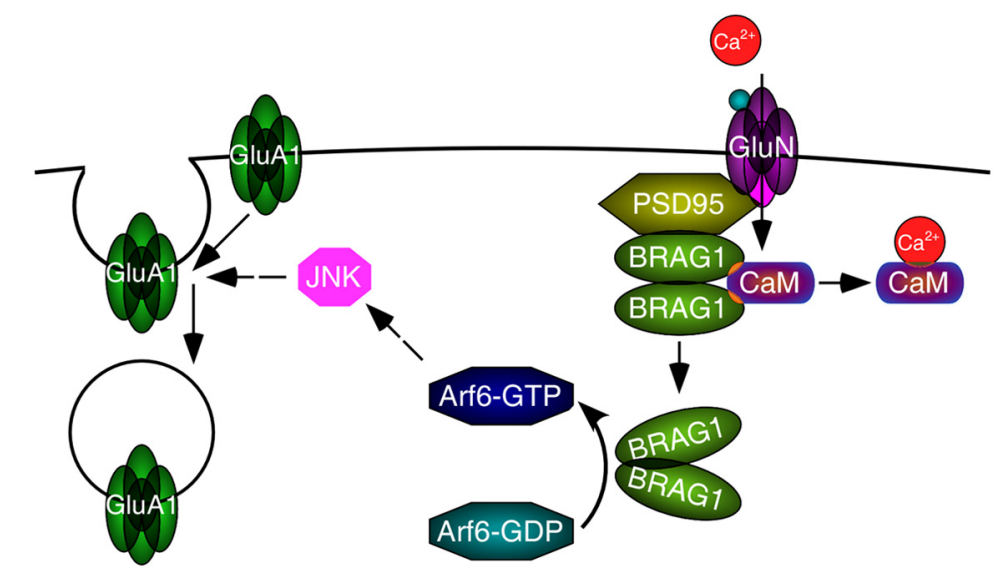

Figure 11. Model for BRAG1-mediated synaptic trafficking of GluA1-containing AMPA-Rs. BRAG1 is anchored at PSDs via its interaction with PSD-95, which also interacts with NMDA receptors. Calcium influx triggered by NMDAR activation causes CaM release from BRAG1, which causes a conformational change leading to enhanced catalytic activity. BRAG1 activates Arf6 which, through downstream activation of JNK, triggers internalization of GluA1-containing AMPA receptors. 
ies have examined the signaling and synaptic mechanisms for two other X-linked mental disorders, oligophrenin-1-associated $\mathrm{X}$-linked mental retardation and fragile X syndrome. Loss of function of oligophrenin-1 is believed to be responsible for the cognitive impairment associated with X-linked mental retardation (Billuart et al., 1998), and recent evidence shows that oligophrenin-1 signals synaptic removal of GluA2-containing AMPA-Rs in a synaptic activity-dependent manner (Nadif Kasri et al., 2009, 2011). In FMR1 knock-out mice, a mouse model for fragile X syndrome (DutchBelgian Fragile X Consortium, 1994), mGluA-dependent LTD is modestly upregulated by $\sim 10-15 \%$, whereas NMDA-R-dependent LTP is considerably reduced (by $\sim 50 \%$ ) in the knock-out animals (Pfeiffer and Huber, 2009; Stornetta and Zhu, 2011). The increased mGluA-dependent LTD is mediated by enhanced Arc signaling (Waung et al., 2008), which controls p38 MAPK-mediated synaptic removal of GluA2-containing AMPA-Rs (Bolshakov et al., 2000; Rial Verde et al., 2006). Exaggerated mGluR signaling seems responsible for several syndromic features of fragile X, including the altered ocular dominance plasticity, seizure, and passive avoidance (Krueger and Bear, 2011). The defect in long-term potentiation (LTP) is due to the selective impairment of signal transduction between Ras and PI3K that abolishes synaptic delivery of GluA1containing AMPA-Rs ( $\mathrm{Hu}$ et al., 2008). This deficient LTP is responsible for the impaired active, high-level associative learning associated with fragile X (Zhao et al., 2005; Hayashi et al., 2007), which is consistent with the finding that synaptic trafficking of GluA1-containing AMPA-Rs is essential for experience-dependent synaptic plasticity and associative learning (Qin et al., 2005; Rumpel et al., 2005; McCormack et al., 2006; Hu et al., 2007; Matsuo et al., 2008; Kielland et al., 2009; Zhu, 2009). Here, we report that BRAG1Arf6 regulates the JNK-mediated synaptic removal of GluA1containing AMPA-Rs (Fig. 11). Moreover, BRAG1 mutations associated with nonsyndromic X-linked mental retardation impair both JNK signaling and synaptic trafficking of GluA1-, but not GluA2-containing AMPA-Rs. These results thus provide the first evidence that dysregulation of JNK signaling and synaptic removal of GluA1-containing AMPA-Rs may also lead to X-linked mental retardation, and provide a new mechanistic explanation for how mutations that either inhibit or enhance Arf6 activity may all result in nonsyndromic X-linked mental disability.

\section{References}

Anggono V, Huganir RL (2012) Regulation of AMPA receptor trafficking and synaptic plasticity. Curr Opin Neurobiol 22:461-469.

Bennett BL, Sasaki DT, Murray BW, O'Leary EC, Sakata ST, Xu W, Leisten JC, Motiwala A, Pierce S, Satoh Y, Bhagwat SS, Manning AM, Anderson DW (2001) SP600125, an anthrapyrazolone inhibitor of Jun N-terminal kinase. Proc Natl Acad Sci U S A 98:13681-13686.

Billuart P, Bienvenu T, Ronce N, des Portes V, Vinet MC, Zemni R, Roest Crollius H, Carri é A, Fauchereau F, Cherry M, Briault S, Hamel B, Fryns JP, Beldjord C, Kahn A, Moraine C, Chelly J (1998) Oligophrenin-1 encodes a rhoGAP protein involved in X-linked mental retardation. Nature 392:923-926.

Bolshakov VY, Carboni L, Cobb MH, Siegelbaum SA, Belardetti F (2000) Dual MAP kinase pathways mediate opposing forms of long-term plasticity at CA3-CA1 synapses. Nat Neurosci 3:1107-1112.

Burkhard P, Stetefeld J, Strelkov SV (2001) Coiled coils: a highly versatile protein folding motif. Trends Cell Biol 11:82-88.

Casanova JE (2007) Regulation of Arf activation: the Sec7 family of guanine nucleotide exchange factors. Traffic 8:1476-1485.

Chen EH, Pryce BA, Tzeng JA, Gonzalez GA, Olson EN (2003) Control of myoblast fusion by a guanine nucleotide exchange factor, loner, and its effector ARF6. Cell 114:751-762.

Choi S, Ko J, Lee JR, Lee HW, Kim K, Chung HS, Kim H, Kim E (2006) ARF6 and EFA6A regulate the development and maintenance of dendritic spines. J Neurosci 26:4811-4819.
Costa RM, Silva AJ (2003) Mouse models of neurofibromatosis type I: bridging the GAP. Trends Mol Med 9:19-23.

Donaldson JG, Jackson CL (2011) ARF family G proteins and their regulators: roles in membrane transport, development and disease. Nat Rev Mol Cell Biol 12:362-375.

Dosemeci A, Makusky AJ, Jankowska-Stephens E, Yang X, Slotta DJ, Markey SP (2007) Composition of the synaptic PSD-95 complex. Mol Cell Proteomics 6:1749-1760.

D'Souza-Schorey C, Chavrier P (2006) ARF proteins: roles in membrane traffic and beyond. Nat Rev Mol Cell Biol 7:347-358.

Dutch-Belgian Fragile X Consortium (1994) Fmrl knockout mice: a model to study fragile X mental retardation. The Dutch-Belgian Fragile X Consortium. Cell 78:23-33.

Fukaya M, Kamata A, Hara Y, Tamaki H, Katsumata O, Ito N, Takeda S, Hata Y, Suzuki T, Watanabe M, Harvey RJ, Sakagami H (2011) SynArfGEF is a guanine nucleotide exchange factor for Arf6 and localizes preferentially at post-synaptic specializations of inhibitory synapses. J Neurochem 116:1122-1137.

Hayashi ML, Rao BS, Seo JS, Choi HS, Dolan BM, Choi SY, Chattarji S, Tonegawa S (2007) Inhibition of p21-activated kinase rescues symptoms of fragile $\mathrm{X}$ syndrome in mice. Proc Natl Acad Sci U S A 104:11489-11494.

Hernández-Deviez DJ, Casanova JE, Wilson JM (2002) Regulation of dendritic development by the ARF exchange factor ARNO. Nat Neurosci 5:623-624.

Hernández-Deviez DJ, Roth MG, Casanova JE, Wilson JM (2004) ARNO and ARF6 regulate axonal elongation and branching through downstream activation of phosphatidylinositol 4-phosphate 5-kinase alpha. Mol Biol Cell 15:111-120.

Hu H, Real E, Takamiya K, Kang MG, Ledoux J, Huganir RL, Malinow R (2007) Emotion enhances learning via norepinephrine regulation of AMPA-receptor trafficking. Cell 131:160-173.

Hu H, Qin Y, Bochorishvili G, Zhu Y, van Aelst L, Zhu JJ (2008) Ras signaling mechanisms underlying impaired GluR1-dependent plasticity associated with fragile X syndrome. J Neurosci 28:7847-7862.

Hussain NK, Hsin H, Huganir RL, Sheng M (2010) MINK and TNIK differentially act on Rap2-mediated signal transduction to regulate neuronal structure and AMPA receptor function. J Neurosci 30:14786-14794.

Jordan BA, Fernholz BD, Boussac M, Xu C, Grigorean G, Ziff EB, Neubert TA (2004) Identification and verification of novel rodent postsynaptic density proteins. Mol Cell Proteomics 3:857-871.

Kelkar N, Gupta S, Dickens M, Davis RJ (2000) Interaction of a mitogenactivated protein kinase signaling module with the neuronal protein JIP3. Mol Cell Biol 20:1030-1043.

Kerchner GA, Nicoll RA (2008) Silent synapses and the emergence of a postsynaptic mechanism for LTP. Nat Rev Neurosci 9:813-825.

Kessels HW, Malinow R (2009) Synaptic AMPA receptor plasticity and behavior. Neuron 61:340-350.

Kielland A, Bochorishvili G, Corson J, Zhang L, Rosin DL, Heggelund P, Zhu JJ (2009) Activity patterns govern synapse-specific AMPA-R trafficking between deliverable and synaptic pools. Neuron 62:84-101.

Kolleker A, Zhu JJ, Schupp BJ, Qin Y, Mack V, Borchardt T, Köhr G, Malinow R, Seeburg PH, Osten P (2003) Glutamatergic plasticity by synaptic delivery of GluR-B(long)-containing AMPA receptors. Neuron 40:1199-1212.

Krauss M, Kinuta M, Wenk MR, De Camilli P, Takei K, Haucke V (2003) ARF6 stimulates clathrin/AP-2 recruitment to synaptic membranes by activating phosphatidylinositol phosphate kinase type Igamma. J Cell Biol 162:113-124.

Krueger DD, Bear MF (2011) Toward fulfilling the promise of molecular medicine in fragile X syndrome. Annu Rev Med 62:411-429.

Lee JC, Kassis S, Kumar S, Badger A, Adams JL (1999) p38 mitogenactivated protein kinase inhibitors-mechanisms and therapeutic potentials. Pharmacol Ther 82:389-397.

Malenka RC, Kauer JA, Zucker RS, Nicoll RA (1988) Postsynaptic calcium is sufficient for potentiation of hippocampal synaptic transmission. Science 242:81-84.

Matsuo N, Reijmers L, Mayford M (2008) Spine-type-specific recruitment of newly synthesized AMPA receptors with learning. Science 319:1104-1107.

McCormack SG, Stornetta RL, Zhu JJ (2006) Synaptic AMPA receptor exchange maintains bidirectional plasticity. Neuron 50:75-88. 
Miyazaki H, Yamazaki M, Watanabe H, Maehama T, Yokozeki T, Kanaho Y (2005) The small GTPase ADP-ribosylation factor 6 negatively regulates dendritic spine formation. FEBS Lett 579:6834-6838.

Montagnac G, Sibarita JB, Loubéry S, Daviet L, Romao M, Raposo G, Chavrier P (2009) ARF6 Interacts with JIP4 to control a motor switch mechanism regulating endosome traffic in cytokinesis. Curr Biol 19:184-195.

Murphy JA, Jensen ON, Walikonis RS (2006) BRAG1, a Sec7 domaincontaining protein, is a component of the postsynaptic density of excitatory synapses. Brain Res 1120:35-45.

Myers KR, Casanova JE (2008) Regulation of actin cytoskeleton dynamics by Arf-family GTPases. Trends Cell Biol 18:184-192.

Nadif Kasri N, Nakano-Kobayashi A, Malinow R, Li B, Van Aelst L (2009) The Rho-linked mental retardation protein oligophrenin-1 controls synapse maturation and plasticity by stabilizing AMPA receptors. Genes Dev 23:1289-1302.

Nadif Kasri N, Nakano-Kobayashi A, Van Aelst L (2011) Rapid synthesis of the X-linked mental retardation protein OPHN1 mediates mGluRdependent LTD through interaction with the endocytic machinery. Neuron 72:300-315.

Ongusaha PP, Qi HH, Raj L, Kim YB, Aaronson SA, Davis RJ, Shi Y, Liao JK, Lee SW (2008) Identification of ROCK1 as an upstream activator of the JIP-3 to JNK signaling axis in response to UVB damage. Science Signaling 1:ra14.

Peng J, Kim MJ, Cheng D, Duong DM, Gygi SP, Sheng M (2004) Semiquantitative proteomic analysis of rat forebrain postsynaptic density fractions by mass spectrometry. J Biol Chem 279:21003-21011.

Pfeiffer BE, Huber KM (2009) The state of synapses in fragile X syndrome. Neuroscientist 15:549-567.

Qin Y, Zhu Y, Baumgart JP, Stornetta RL, Seidenman K, Mack V, van Aelst L, Zhu JJ (2005) State-dependent Ras signaling and AMPA receptor trafficking. Genes Dev 19:2000-2015.

Rhoads AR, Friedberg F (1997) Sequence motifs for calmodulin recognition. FASEB J 11:331-340.

Rial Verde EM, Lee-Osbourne J, Worley PF, Malinow R, Cline HT (2006) Increased expression of the immediate-early gene arc/arg3.1 reduces AMPA receptor-mediated synaptic transmission. Neuron 52:461-474.

Rumpel S, LeDoux J, Zador A, Malinow R (2005) Postsynaptic receptor trafficking underlying a form of associative learning. Science 308:83-88.

Sakagami H, Sanda M, Fukaya M, Miyazaki T, Sukegawa J, Yanagisawa T, Suzuki T, Fukunaga K, Watanabe M, Kondo H (2008) IQ-ArfGEF/ BRAG1 is a guanine nucleotide exchange factor for Arf6 that interacts with PSD-95 at postsynaptic density of excitatory synapses. Neurosci Res 60:199-212.

Santy LC, Casanova JE (2001) Activation of ARF6 by ARNO stimulates ep- ithelial cell migration through downstream activation of both Rac1 and phospholipase D. J Cell Biol 154:599-610.

Scholz R, Berberich S, Rathgeber L, Kolleker A, Köhr G, Kornau HC (2010) AMPA receptor signaling through BRAG2 and Arf6 critical for long-term synaptic depression. Neuron 66:768-780.

Shoubridge C, et al. (2010) Mutations in the guanine nucleotide exchange factor gene IQSEC2 cause nonsyndromic intellectual disability. Nat Genet 42:486-488.

Stornetta RL, Zhu JJ (2011) Ras and Rap signaling in synaptic plasticity and mental disorders. Neuroscientist 17:54-78.

Suzuki A, Arikawa C, Kuwahara Y, Itoh K, Watanabe M, Watanabe H, Suzuki T, Funakoshi Y, Hasegawa H, Kanaho Y (2010) The scaffold protein JIP3 functions as a downstream effector of the small GTPase ARF6 to regulate neurite morphogenesis of cortical neurons. FEBS Lett 584:2801-2806.

Thomas GM, Huganir RL (2004) MAPK cascade signalling and synaptic plasticity. Nat Rev Neurosci 5:173-183.

Traynelis SF, Wollmuth LP, McBain CJ, Menniti FS, Vance KM, Ogden KK, Hansen KB, Yuan H, Myers SJ, Dingledine R (2010) Glutamate receptor ion channels: structure, regulation, and function. Pharmacol Rev 62:405-496.

Waung MW, Pfeiffer BE, Nosyreva ED, Ronesi JA, Huber KM (2008) Rapid translation of Arc/Arg3.1 selectively mediates mGluR-dependent LTD through persistent increases in AMPAR endocytosis rate. Neuron 59:84-97.

Yang H, Courtney MJ, Martinsson P, Manahan-Vaughan D (2011) Hippocampal long-term depression is enhanced, depotentiation is inhibited and long-term potentiation is unaffected by the application of a selective c-Jun N-terminal kinase inhibitor to freely behaving rats. Eur J Neurosci 33:1647-1655.

Zhang H, Webb DJ, Asmussen H, Horwitz AF (2003) Synapse formation is regulated by the signaling adaptor GIT1. J Cell Biol 161:131-142.

Zhao MG, Toyoda H, Ko SW, Ding HK, Wu LJ, Zhuo M (2005) Deficits in trace fear memory and long-term potentiation in a mouse model for fragile X syndrome. J Neurosci 25:7385-7392.

Zhu JJ (2009) Activity level-dependent synapse-specific AMPA receptor trafficking regulates transmission kinetics. J Neurosci 29:6320-6335.

Zhu JJ, Esteban JA, Hayashi Y, Malinow R (2000) Postnatal synaptic potentiation: delivery of GluR4-containing AMPA receptors by spontaneous activity. Nat Neurosci 3:1098-1106.

Zhu JJ, Qin Y, Zhao M, Van Aelst L, Malinow R (2002) Ras and Rap control AMPA receptor trafficking during synaptic plasticity. Cell 110:443-455.

Zhu Y, Pak D, Qin Y, McCormack SG, Kim MJ, Baumgart JP, Velamoor V, Auberson YP, Osten P, van Aelst L, Sheng M, Zhu JJ (2005) Rap2-JNK removes synaptic AMPA receptors during depotentiation. Neuron 46: 905-916. 